JOURNAL OF BUSINESS

and entrepreneurial

studies

\title{
Propuesta de un complejo turístico en la Parroquia Chanduy, Provincia de Santa Elena
}

\section{Proposal for a tourist complex in Chanduy Parish, Santa Elena Province}

Ing. Félix Olivero Sánchez, Mgs

Universidad Davis California

Los Angeles - Estados Unidos

felixsanchez@hotmail.com

ORCID https://orcid.org/0000-0001-9967-2242

Ing. Marlene Montesdeoca Peralta, Mgs

Universidad Politécnica de Madrid

Madrid - España

marco.suriagas@ug.edu.ec

ORCID https://orcid.org/0000-0002-0159-6104

Recibido 5 julio 2017 - Aceptado 23 noviembre - 2017 


\section{Resumen}

La investigación tiene como objetivo proponer un complejo Turístico en Chanduy, provincia de Santa Elena. Realizado el diagnostico situacional se pudo determinar que la Parroquia Chanduy, cuenta con elementos naturales y autóctonos para atraer turismo rentable y beneficioso, generando fuentes de empleo a los comuneros, en el primer capítulo denominado estudio de mercado muestra al lector el comportamiento de los clientes potenciales, la demanda y oferta del sector económico al cual pertenece la entidad, definir las condiciones que la organización debe tener para ser competente en el mercado y la estrategia comercial, el capítulo del estudio técnico permite determinar los recursos necesarios para el desarrollo del proyecto, sus respectivos costos operacionales y la ubicación del complejo, estudios organizacionales y legales los cuales permitirán diseñar la estructura, políticas de la empresa teniendo en cuenta los demuestra la factibilidad del proyecto utilizando indicadores financieros tales como el VAN, TIR.

Palabras Clave: Complejo turístico, Estudio de mercado, Factibilidad.

\section{Summary}

The research aims to propose a Tourist complex in Chanduy, province of Santa Elena. Once the situational diagnosis was made, it was possible to determine that Chanduy Parish has natural and native elements to attract profitable and beneficial tourism, generating employment sources for the community members, in the first chapter called a market study shows the reader the behavior of potential clients, the demand and supply of the economic sector to which the entity belongs, to define the conditions that the organization must have to be competent in the market and the commercial strategy, the chapter of the technical study allows to determine the necessary resources for the development of the project, its respective operational costs and the location of the complex, organizational and legal studies which will allow to design the structure, policies of the company taking into account demonstrates the feasibility of the project using financial indicators such as VAN, TIR.

Keywords: Tourist complex, Market study, Feasibility.

\section{INTRODUCCIÓN}

En Ecuador el desarrollo turístico inicia en los años 50, y es principalmente en las últimas tres décadas en que empieza a establecerse como actividad económica y socio cultural, convirtiéndose en uno de los rubros de generación de divisas más significativas, de tal manera que se ha convertido en un sector estratégico para el desarrollo del país, representando la tercera actividad (5.78\%) en la escala de ingresos no petroleros, luego del banano $(10.13 \%)$ y el camarón $(9.99 \%)$ (Mintur, Ministerio de Turismo del Ecuador, 2015). Además es considerado como uno de los 12 países con mayor diversidad del mundo, pluricultural y poseedor de diversidades de ecosistemas en un territorio particularmente pequeño, con buenas condiciones de conectividad interna y ubicada estratégicamente, es decir que posee condiciones excepcionales para poder proyectar su desarrollo turístico y de esta forma 
ampliar su economía. (Vargas, Fuentes, \& Ocaña, 2014)

Actualmente la provincia de Santa Elena es la tercera provincia con mayor acogida de turistas nacionales e internacionales (Mintur, 2015), pero aún existen sectores que no han sido aprovechados, dentro de estos sectores nos encontramos con la parroquia Chanduy que es un lugar con alto potencial turístico (Guillen Muñoz, 2015), pero debido a la carencia de información, conocimiento en turismo e incentivos para la inversión ha generado que no existan infraestructuras destinada para acoger turistas.

Para lo cual se ha tomado como tema el "estudio de factibilidad para la creación de un complejo turístico", conservando y recuperando los recursos naturales para el buen uso productivo de los mismos de forma responsable y consciente promoviendo el desarrollo sostenible y sustentable para alcanzar el buen vivir, tomando al turismo como eje fundamental para la activación económica y generación de empleo.

Para ello dentro del complejo turístico se ofrecerán paquetes turísticos que incluirán transporte, hospedaje, alimentación, entretenimiento, guías turísticos, asimismo se pretende colaborar con la preservación del medio ambiente, con el fin de conservar la belleza natural de la región, ya que la existencia de estos ecosistemas favorecen la vocación pesquera y turística de Chanduy, de manera que con un adecuado uso de sus recursos se convertirá en un potencial turístico para el desarrollo económico y social de la parroquia.

Debido a que existen pocos espacios como un complejo turístico en la parroquia, se espera que esta investigación sirva como base de futuros proyectos que tengan como objetivo convertirla en un destino turístico, para ello se ha realizado encuestas $y$ entrevistas a personas de la comunidad, logrando conocer la demanda turística nacional y extranjera que visita el balneario.

\section{Materiales y métodos}

El tipo de investigación es descriptiva y explicativa pues logra caracterizar un objeto de estudio o una situación concreta, señalar sus características y propiedades. Sirve para ordenar agrupar o sistematizar los objetivos involucrados en el trabajo indagatorio. Mediante la investigación descriptiva se interpreta los fenómenos básicos del problema para así desarrollar estrategias adecuadas con la finalidad de mejorar la calidad de vida en Chanduy.

Esta investigación se apoya en informaciones que provienen entre otras, de entrevistas, cuestionarios, encuestas y observaciones. Este proceso se aplicó para conocer la necesidad de la creación de un complejo turístico y a su vez constatar el conocimiento de la población sobre las actividades turísticas que se podrían desarrollar en Chanduy. Los dirigentes comunales ayudaron en la investigación para obtener un levantamiento turístico de la zona y así poder destacar mayores oportunidades 
de turismo comunitario en futuras ocasiones.

Apoyándose en fuentes de carácter documental, esto es, en documentos de cualquier especie. Investigación bibliográfica: se basa en consulta de libros, Tradiciones, Costumbres, Folclor y Creencias de mi Pueblo: Chanduy. Santa Elena, diccionario histórico peninsular - guía didáctica de la península Santa Elena - morro. Santa Elena. Investigación hemerográfica: se basa en artículos o ensayos de revistas y periódicos. Resultados Regionales del Turismo. Panorama OMT del turismo internacional, Edicion 2015, Ecuador em cifras, Poblacion Economicamente Activa.

Como método empírico se usó la entrevista, con un instrumento de recolección de información o datos mediante una conversación profesional y formal con la finalidad de adquirir conocimientos del tema de investigación. Por lo regular se obtiene mayor información, ya que si una respuesta ha sido incompleta, se pude realizar otra pregunta que ayude a complementarla

También la encuesta, esta es un instrumento de investigación convencional que se realiza a un segmento de mercado para recolectar información de diversos tópicos con la finalidad de obtener opiniones $\mathrm{o}$ valoración de un fenómeno.

El método de observación consiste en realizar visitas para recabar datos previos a la situación actual de la población a estudiar. Es decir observar la conducta de los turistas al llegar a Chanduy en lugar de basarnos en las respuestas.

La presentación de la investigación se basa en la implementación y diseño de una encuesta a personas de un nivel socio económico medio y medio alto, que permite obtener conocimiento del grupo objetivo, para poder así desarrollar el plan estratégico de marketing. De esta manera, se establecerá el posicionamiento actual del complejo turístico y sus competidores, para entender el estado situacional del producto.

Este análisis, proporcionará información clave para realizar el plan estratégico de Marketing y la futura toma de decisiones. Para determinar el tamaño de la muestra de la población, se consultó al Instituto Nacional de Estadísticas y Censos con el fin de obtener la información exacta de la cantidad de pobladores de la ciudad de Guayaquil dando como resultado lo siguiente:

Se utilizará el método aleatorio simple con una muestra probabilística estratificada debido a que nuestra segmentación fue dirigida a la Provincia del Guayas, ciudad de Guayaquil, con una población de ingresos económicos medio-alto los que nos permitirá obtener datos confiables.

$$
\begin{gathered}
E=Z c\left(\frac{\sigma}{\sqrt{n}}\right) \sqrt{\frac{N-n}{N-1}} \\
0.03=2.17\left(\frac{0.25}{\sqrt{n}}\right) \sqrt{\frac{5,176-n}{5,176-1}}
\end{gathered}
$$




$$
\begin{gathered}
\sqrt{n}=2.17\left(\frac{0.25}{0.0 .3}\right) \sqrt{\frac{5,176-n}{5,175}} \\
\sqrt{n}=18.0833 * \sqrt{\frac{5,176-n}{5,175}} \\
n=327.0069 *\left(\frac{5,176-n}{5,175}\right) \\
5,175 n=1^{\prime} 692587.944 \\
-327.0069 n \\
5,175 n+327.0069 n \\
=1^{\prime} 692587.994 \\
5,502.0069 n=1^{\prime} 692587.994 \\
n=\frac{1^{\prime} 692587.994}{5,502.0069} \\
n=308 \text { habitantes }
\end{gathered}
$$

Con la aplicación de la fórmula se concluye que las encuestas serán aplicadas a 308 personas, y el modelo a seguir será el siguiente:

\section{Resultados}

\section{1.- ¿Cuál es su edad?}

Tabla 1: ¿Cuál es su edad?

\begin{tabular}{ll}
\hline EDAD & SUMA \\
\hline 11 a 18 & 7 \\
18 a 25 & 30 \\
26 a 30 & 71 \\
31 a 40 & 57 \\
41 a 50 & 33 \\
51 o más & 110 \\
Total & $\mathbf{3 0 8}$ \\
\hline
\end{tabular}

Figura 1: ¿Cuál es su edad?

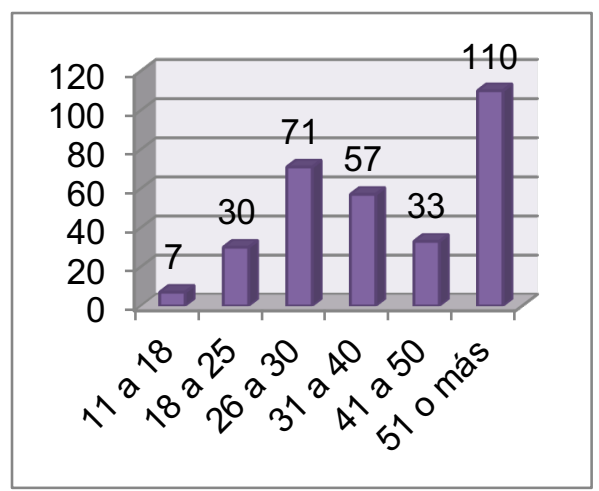

Nota: Autores de la investigación.

Para obtener información acerca de la edad de los consultados se estableció 6 intervalos (Columna 1), de estos el de mayor relevancia es aquel que indica que el $35.7 \%$ de los entrevistados tienen 51 o más años de edad.

\section{2.- ¿Cuál es su sexo?}

Tabla 2: ¿Cuál es su sexo?

\begin{tabular}{ll}
\hline SEXO & SUMA \\
\hline Femenino & 184 \\
Masculino & 124 \\
Total & $\mathbf{3 0 8}$ \\
\hline
\end{tabular}

Figura 2: ¿Cuál es su sexo?

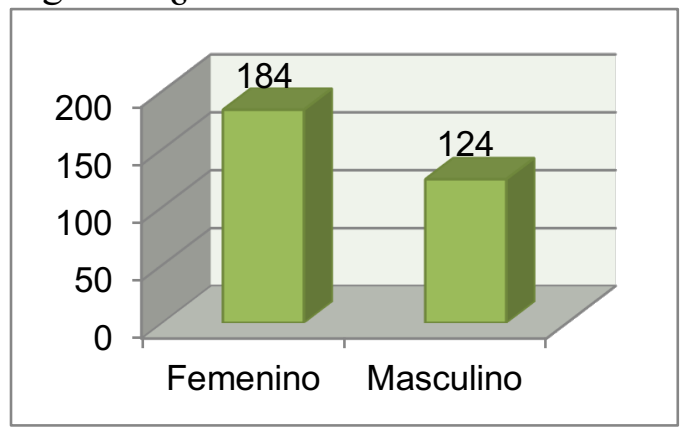

Se puede observar que 124 de los 308 consultados son de sexo masculino, esto corresponde al $40.3 \%$ de la muestra. Y 184 de los 300 consultados son del género femenino, esto corresponde al $59.7 \%$ de la muestra. 
JOURNAL OF BUSINESS

and entrepreneurial

studies

3.- ¿Cuál es su estado civil?

Tabla 3: ¿Cuál es su estado civil?

\begin{tabular}{ll} 
ESTADO CIVIL & TOTAL \\
\hline Casado & 171 \\
Otros & 69 \\
Soltero & 68 \\
Total & $\mathbf{3 0 8}$ \\
\hline
\end{tabular}

Figura 3: ¿Cuál es su estado civil?

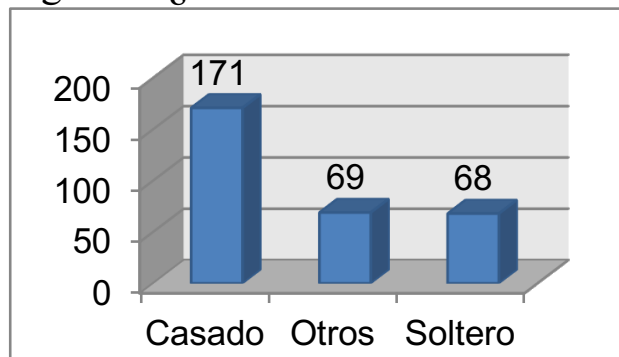

Esta variable registra el estado civil de la persona a la cual se entrevistó, de estas frecuencias se destaca que 171 (55.5\%) de los entrevistados tiene por estado civil el de casado.

4.- ¿Cuál es su nivel de instrucción o formación?

Tabla4 : Nivel de educación

\begin{tabular}{ll}
\hline NIVEL DE EDUCACIÓN & TOTAL. \\
\hline Primaria & 35 \\
Secundaria & 89 \\
Superior & 184 \\
Total & $\mathbf{3 0 8}$ \\
\hline
\end{tabular}

Figura 4: ¿Cuál es su nivel de instrucción?

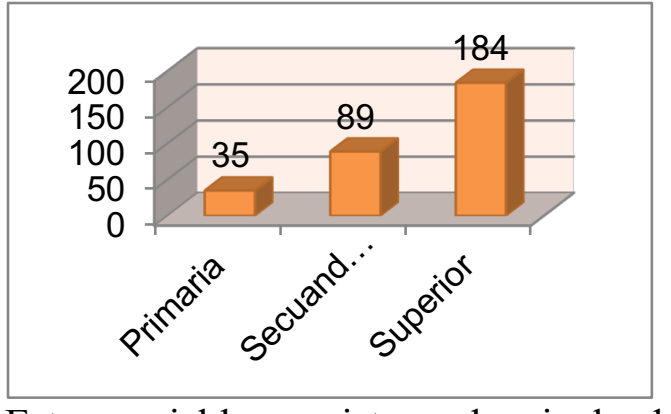

Esta variable registra el nivel de formación de la persona a la cual se entrevistó, de estas frecuencias se destaca que $184 \quad(59,7 \%)$ de los entrevistados tiene nivel superior de formación.

5.- ¿De los siguientes tipos de turismo, cuales Ud. ha realizado?

Tabla5: Tipos de turismo

\begin{tabular}{lllll}
\hline TIP & TURIS & EC & & \\
OS & MO & OT & TURI & TURI \\
DE & DE & UR & SMO & SMO \\
TUR & SOL Y & IS & DE & COM \\
ISM & PLAY & M & AVEN & UNIT \\
O & A & O & TURA & ARIO \\
\hline Tota & & & & \\
l & 227 & 82 & 69 & 60 \\
\hline
\end{tabular}

Figura 5: Tipo de turismo

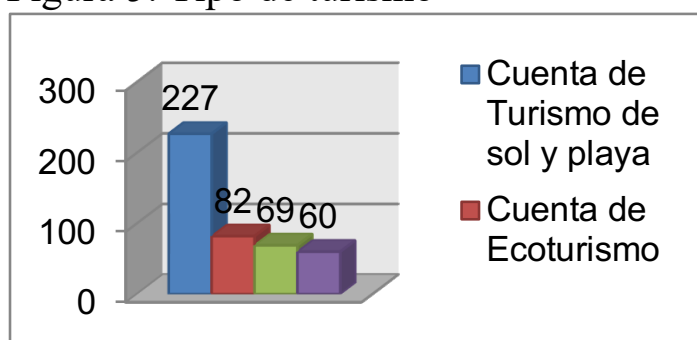

Esta variable registra el número de personas que han realizado al menos un viaje, indicando que $227 \quad(73.7 \%)$ personas han realizado viaje(s) por turismo de sol y playa frente a 82 $(26.6 \%)$ personas que lo han realizado 
por ecoturismo. El $22,40 \%$ de los encuestados realizó turismo de aventura frente al 19,48\% que realizó turismo comunitario.

6.- ¿En qué meses del año prefiere viajar por motivo de realizar turismos?

Tabla 6: Meses de viajes por turismo

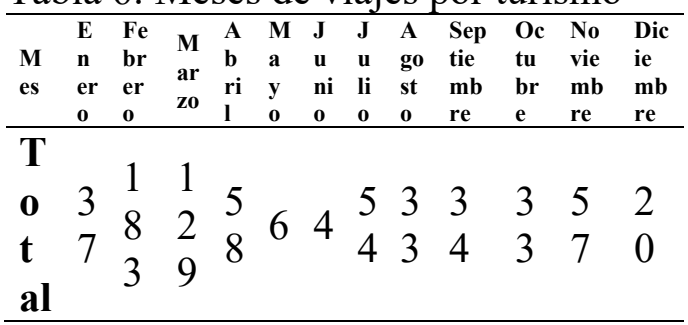

Figura 6: Meses de viajes por turismo

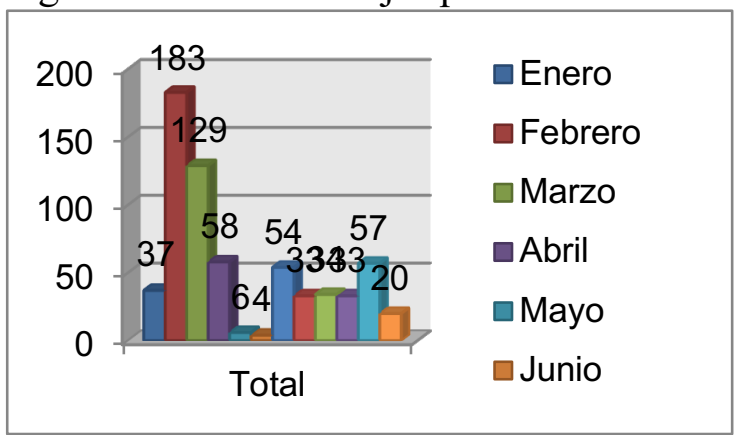

Esta variable registra los meses en que viajan por motivos de turismo los consultados, en cual se indica que el $59,42 \%$ de los individuos que viajan lo hacen en el mes de febrero por motivos de vacaciones y feriado de carnaval, siendo este el porcentaje más alto detectado.

\section{7.- ¿Quiénes participan en el viaje de} turismo?

Tabla 7: Compañía en viajes

\begin{tabular}{llllll}
\hline $\begin{array}{l}\text { COMP } \\
\text { AÑ́́ }\end{array}$ & $\begin{array}{l}\text { AMI } \\
\text { GOS }\end{array}$ & $\begin{array}{l}\text { HIJ } \\
\text { O(S) }\end{array}$ & $\begin{array}{l}\text { PA } \\
\text { PÁ }\end{array}$ & $\begin{array}{l}\text { MÁ́ } \\
\text { MÁ }\end{array}$ & $\begin{array}{l}\text { OT } \\
\text { RO } \\
\text { S }\end{array}$ \\
\hline & & & 11 & & \\
Total & 178 & 197 & 8 & 114 & 43 \\
\hline
\end{tabular}

Figura 7: Compañía en viajes

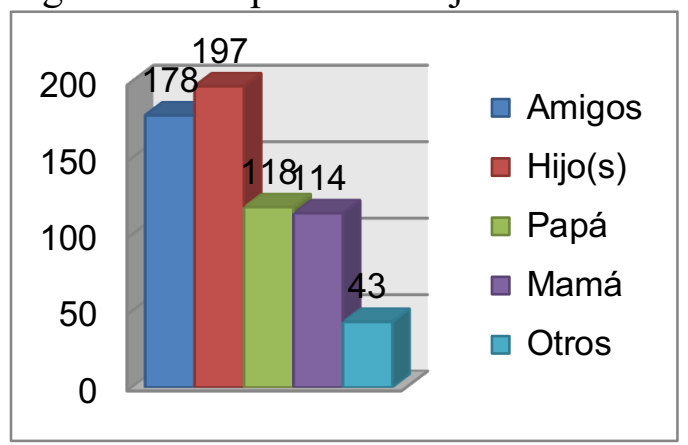

Se sondeó además sobre las personas que participarían en el viaje de turismo, la información más relevante indica que más de la mitad (63.96\%) de las personas consultadas consideran viajar con sus hijos frente a otro porcentaje destacado de $(57.79 \%)$ consultados que indicaron que viajarían con sus amigos.

\section{8.- ¿Cuál es valor diario aproximado} que Ud. estaría dispuesto a gastar?

Tabla 8: Valor a gastar

\begin{tabular}{ll}
\hline RANGO DE GASTOS & TOTAL \\
\hline Menor a $\$ 50$ & 100 \\
\$50 a \$100 & 167 \\
\$151 o más & 5 \\
\$101 a \$1150 & 36 \\
Total & $\mathbf{3 0 8}$ \\
\hline
\end{tabular}

Figura 8: Valor a gastar 
JOURNAL OF BUSINESS

and entrepreneurial

studies

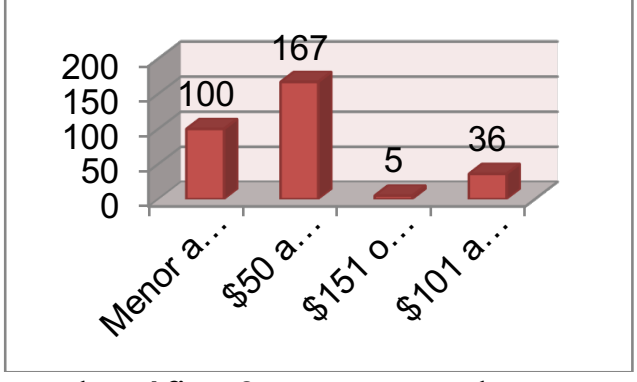

En el Gráfico 8 se muestran los porcentajes de los consultados que seleccionaron los respectivos intervalos de dinero dispuestos a gastar aproximadamente en un día. Arrojando como resultados que el $54.2 \%$ prefiere gastar en un rango de $\$ 50$ a $\$ 100$.

9.- ¿En sus viajes fuera de Guayaquil?, ¿Le interesa conocer la cultura del lugar que visita?

Tabla 9: Cultura

\begin{tabular}{ll}
\hline CULTURA & $\begin{array}{l}\text { CUENTA DE } \\
\text { CULTURA }\end{array}$ \\
\hline No & 4 \\
$\mathrm{Si}$ & 304 \\
Total & $\mathbf{3 0 8}$ \\
\hline
\end{tabular}

Figura 9: Cultura

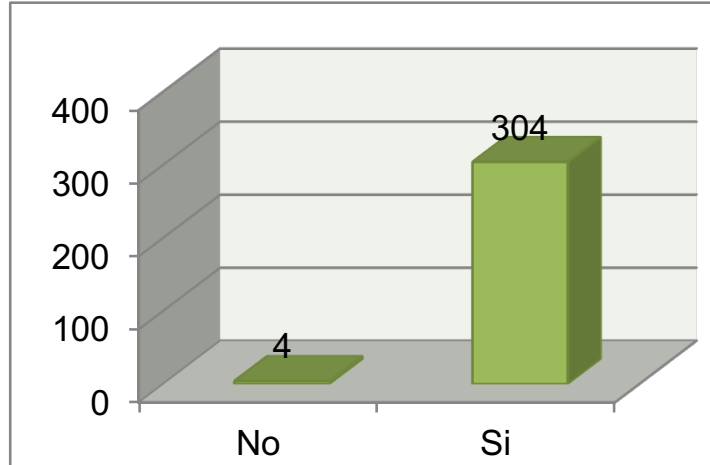

Se consultó además sobre el interés en conocer sobre la cultura del lugar, la información indica que 304 (98,7\%) considera que si debería conocer sobre la cultura del lugar que visita mientras que un $1,3 \%$ de estas personas no lo consideran necesario.

10.- ¿Ha escuchado acerca del turismo comunitario?

Tabla 10: Turismo Comunitario

\begin{tabular}{ll}
\hline TURISMO COMUNITARIO & TOTAL \\
\hline NO & 76 \\
SI & 232 \\
TOTAL & $\mathbf{3 0 8}$ \\
\hline
\end{tabular}

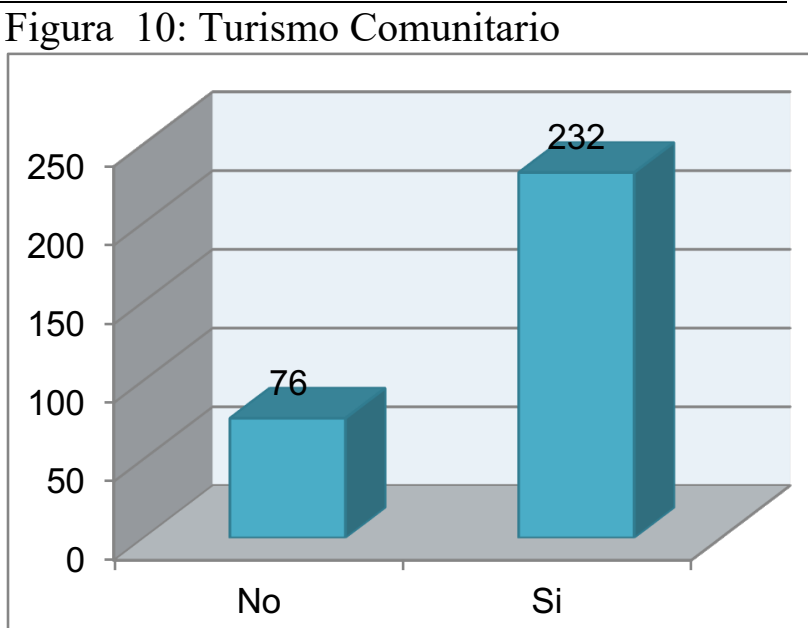

En el Gráfico 10 se hace evidente que más de la mitad $(75.3 \%)$ ha escudo sobre el turismo comunitario.

11.- ¿Qué tan interesado estaría Ud. en realizar un viaje que le permita participar en el estilo de vida de una comunidad?

Tabla 11: Estilo de Vida

\begin{tabular}{ll}
\hline ESTILO DE VIDA EN & \\
COMUNIDAD & Total \\
\hline Interesado & 150 \\
Muy interesado & 152 \\
Poco interesado & 6 \\
Total general & $\mathbf{3 0 8}$ \\
\hline
\end{tabular}


Figura 10: Estilo de vida en comunidad

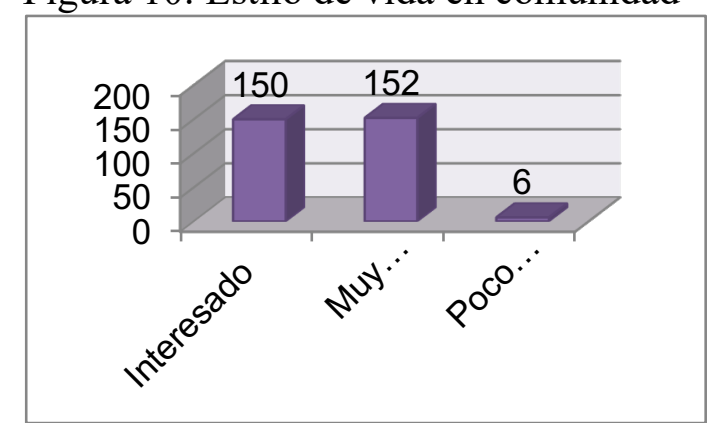

El 49.35\% de la muestra está muy interesado de participar en el estilo de vida de una comunidad, el $48.7 \%$ de las personas están interesados, mientras que el $1.95 \%$ está poco interesado en participar.

12.- ¿Conoce la parroquia Chanduy? Tabla 12: Conoce la parroquia Chanduy

\section{CHANDUY TOTAL}

\begin{tabular}{ll} 
No & 137 \\
$\mathrm{Si}$ & 171 \\
Total & $\mathbf{3 0 8}$ \\
\hline
\end{tabular}

Figura 11: ¿Conoce la parroquia Chanduy

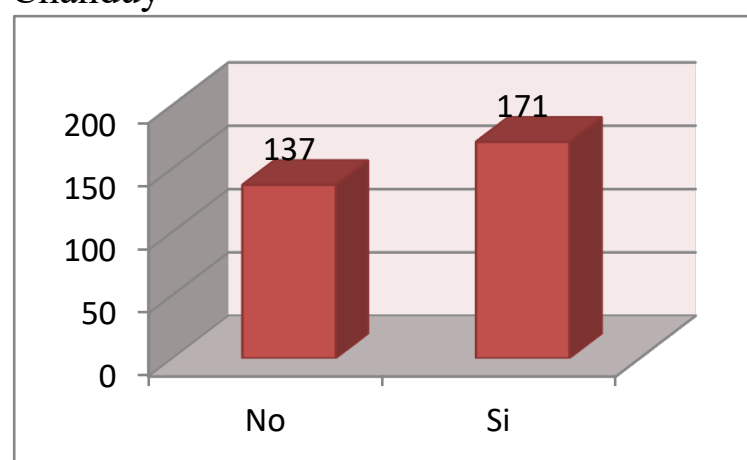

Se cuestionó si las personas que viajan conocen la parroquia Chanduy de la Provincia de Santa. Elena, de los datos recolectados se obtuvo información que indica que el 55,52\% de los consultados conocen Chanduy ubicada en la provincia de Santa Elena y un $44,48 \%$ que no conocen.

13.- ¿Estaría dispuesto a viajar a la parroquia Chanduy en la Provincia de Santa Elena?

Tabla 13: Viaje a Chanduy

\begin{tabular}{ll}
\hline VIAJE A & TOTAL \\
CHANDUY & \\
\hline Interesado & 143 \\
Muy interesado & 161 \\
Poco interesado & 4 \\
Total & $\mathbf{3 0 8}$ \\
\hline
\end{tabular}

Figura 12: Viaje a Chanduy

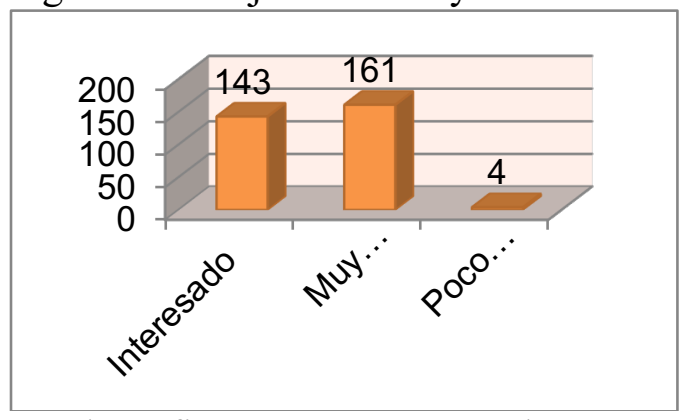

En el Gráfico 13 se muestran el porcentaje $(52.27 \%)$ de los consultados que estarían dispuestos a viajar a la parroquia Chanduy; el $46.43 \%$ estaría interesado y el $1,30 \%$ poco interesado en viajar.

\section{4.- ¿Le gustaría conocer los} atractivos turísticos de la zona? Tabla 14: Conocer atractivos de Chanduy

\begin{tabular}{ll}
\hline CONOCER & TOTAL \\
ATRACTIVOS & 6 \\
\hline No & 302 \\
Si & $\mathbf{3 0 8}$ \\
Total & \\
\hline
\end{tabular}


Figura 13: Conocer atractivos de Chanduy

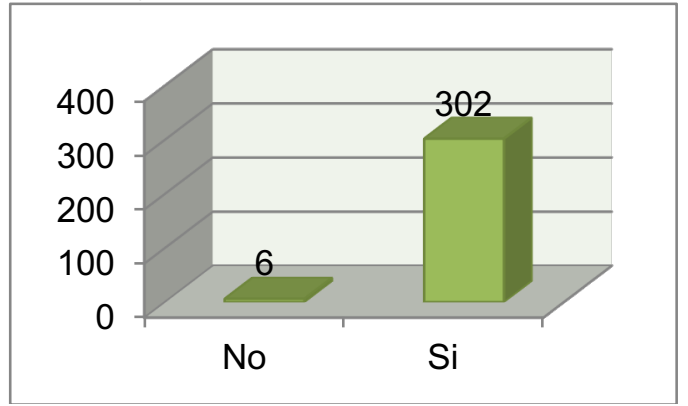

En el Gráfico 12 se hace evidente que la mayoría $(98.1 \%)$ le gustaría conocer los atractivos de la zona.

15.- ¿De los siguientes atractivos turísticos, cuál estaría dispuesto a visitar?

Tabla 15: Atractivos turísticos a visitar

\begin{tabular}{llllll}
\hline AT & BOS & PL & PLA & MU & MIR \\
RA & QUE & AY & DE & O & ADO \\
CTI & DE & A & RA & CE & DEL \\
VO & LA & DE & CO & RR & CER \\
S & COM & C & MU & O & RO \\
TU & UNA & H & NA & RE & TUG \\
RÍS & PEC & AN & EL & AL & ADU \\
TIC & HIC & DU & REA & AL & AJA \\
OS & HE & Y & L & TO & \\
\hline & & 24 & & & \\
Sí & 165 & 1 & 163 & 188 & 143 \\
No & 52 & 2 & 10 & 8 & 22 \\
Vac & & & & & \\
ías & 91 & 65 & 135 & 112 & 143 \\
Tot & & $\mathbf{3 0}$ & & & \\
al & $\mathbf{3 0 8}$ & $\mathbf{8}$ & $\mathbf{3 0 8}$ & $\mathbf{3 0 8}$ & $\mathbf{3 0 8}$ \\
\hline
\end{tabular}

Figura 14: Atractivos turísticos a visitar

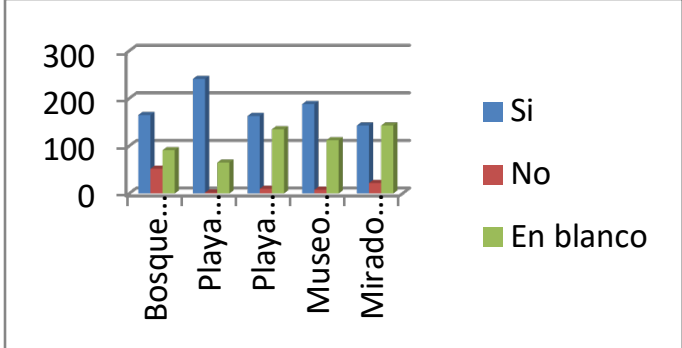

Esta variable registra los atractivos turísticos que las personas estarían dispuestas a visitar, indicando que 165 (53.57\%) personas visitarían el Bosque de la Comuna Pechiche; 241 (78.25\%) personas visitaría la Playa de Chanduy; $163(52.92 \%)$ visitaría la Playa de la Comuna El Real; 188 (61.04\%) visitaría el Museo Cerro Real Alto frente a 143 (46.43\%) personas irían al Mirador del Cerro Tugaduaja.

16.- ¿Estaría dispuesto a visitar y/o hospedarse en un complejo turístico en Chanduy, Provincia de Santa Elena?

Tabla 16: Hospedaje en Chanduy

\begin{tabular}{ll}
\hline HOSPEDAJE & TOTAL \\
\hline No & 4 \\
$\mathrm{Si}$ & 304 \\
Total & $\mathbf{3 0 8}$ \\
\hline Gráfico & $15:$ Hospedaje en Chanduy
\end{tabular}

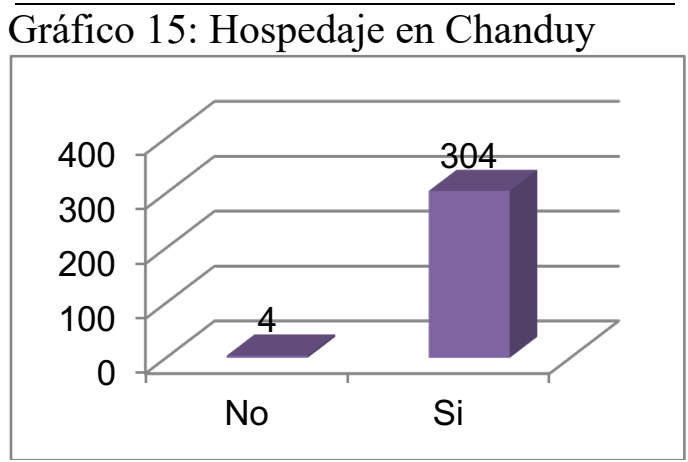


A los consultados que viajan se les preguntó si estarían dispuestos a hospedarse en un complejo turístico en Chanduy, Provincia de Santa Elena, sus en el complejo, la variable que recopila esta información es tiempo de permanencia. En el Gráfico 17 se visualiza el tiempo (días), que los

\begin{tabular}{lllllllll}
\hline Actividades & $\begin{array}{l}\text { Dormir en casa } \\
\text { de una familia } \\
\text { de la comunidad }\end{array}$ & Pescar & $\begin{array}{l}\text { Snork } \\
\text { el }\end{array}$ & $\begin{array}{l}\text { Probar } \\
\text { comida } \\
\text { típica }\end{array}$ & $\begin{array}{l}\text { Aprender a } \\
\text { preparar } \\
\text { comida típica }\end{array}$ & $\begin{array}{l}\text { Realizar } \\
\text { una } \\
\text { caminata }\end{array}$ & $\begin{array}{l}\text { Jugar vóley } \\
\text { o fútbol }\end{array}$ & $\begin{array}{l}\text { Actividades de } \\
\text { agricultura }\end{array}$ \\
\hline $\begin{array}{l}\text { Poco } \\
\text { Interesado }\end{array}$ & 64 & 50 & 64 & 18 & 26 & 48 & 44 & 98 \\
Interesado & 182 & 98 & 76 & 66 & 86 & 90 & 100 & 58 \\
$\begin{array}{l}\text { Muy } \\
\text { Interesado }\end{array}$ & 23 & 12 & 17 & 54 & 34 & 85 & 28 & 12 \\
En blanco & 39 & 148 & 151 & 170 & 162 & 85 & 136 & 140 \\
Total & $\mathbf{3 0 8}$ & $\mathbf{3 0 8}$ & $\mathbf{3 0 8}$ & $\mathbf{3 0 8}$ & $\mathbf{3 0 8}$ & $\mathbf{3 0 8}$ & $\mathbf{3 0 8}$ & $\mathbf{3 0 8}$ \\
\hline
\end{tabular}

respuestas produjeron la siguiente información presentada en el gráfico. En el Gráfico 16 se muestran el porcentaje $(98,7 \%)$ de los consultados que estarían dispuestos a hospedarse en el complejo.

\section{7.- ¿Cuántos días estaría dispuesto a} permanecer en el complejo turístico?

Tabla 17: Tiempo de hospedaje

\begin{tabular}{ll}
\hline TIEMPO DE & TOTAL \\
HOSPEDAJE & 19 \\
\hline 1 día & 136 \\
2 días & 153 \\
3 o más & $\mathbf{3 0 8}$ \\
Total & \\
\hline
\end{tabular}

Figura 16: Tiempo de hospedaje

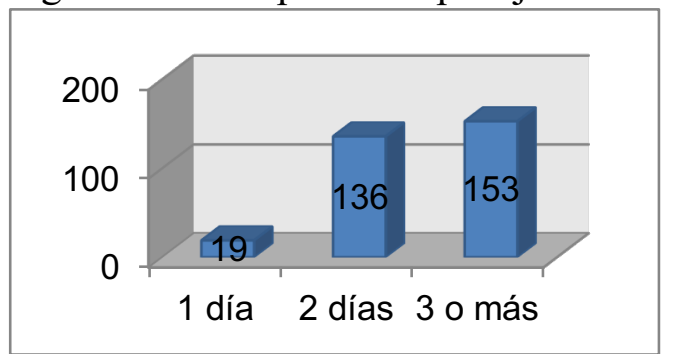

A las personas se les preguntó el número de días que permanecerían hospedados consultados permanecerían hospedados en el complejo de estudio, dichos porcentajes corresponden al $49.68 \%$ se hospedaría 3 o más días el $44.16 \%$ se hospedaría 2 días y el $6.17 \%$ se hospedaría 1 día.

\section{8.- ¿Qué tan interesado estaría Ud.} en realizar las siguientes actividades?

Tabla 18: Actividades a realizar dentro del complejo turístico

Figura 17: Actividades a realizar dentro del complejo turístico

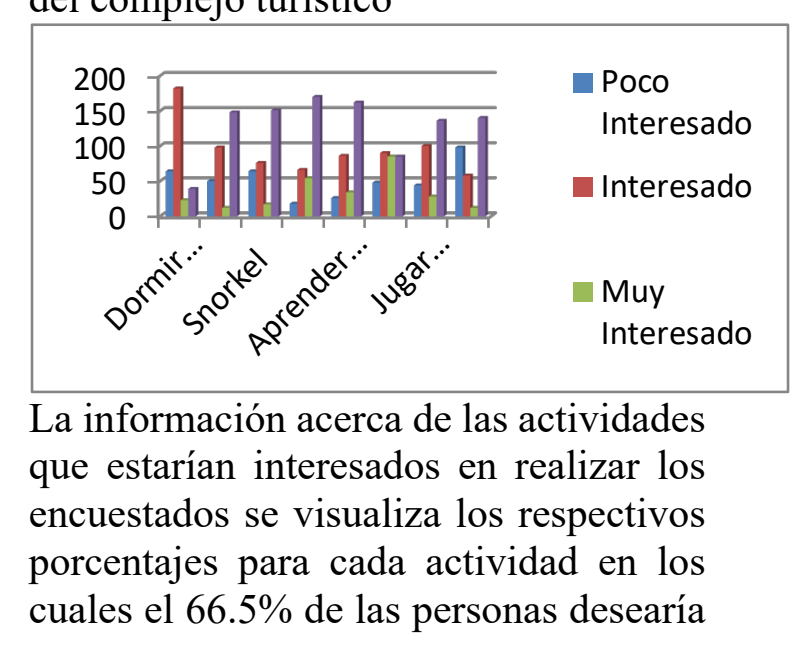


dormir en casa de una familia de la comunidad; el $35.7 \%$ de las personas desearía pescar; el $30.2 \%$ desearía practicar Snorkel; el 39\% desearía probar la comida típica, el 39\% manifiesta que aprendería a preparar la comida típica, mientras que el $56.8 \%$ estaría dispuesto a realizar una caminata por el sector, el $41.6 \%$ practicaría futbol o vóley con los lugareños, finalmente el $22.7 \%$ realizaría actividades de agricultura de la zona.

19.- ¿Qué tan importante le parece contar con un guía que explique las tradiciones, costumbres e historia del lugar, con el fin de profundizar en el aprendizaje de la cultura de la comunidad visitada?

Tabla 19: Importancia de contar con guía turístico

\begin{tabular}{ll}
\hline GUÍA & TOTAL \\
\hline Importante & 73 \\
Muy importante & 235 \\
Total & $\mathbf{3 0 8}$ \\
\hline
\end{tabular}

Figura 18: Importancia de contar con guía turístico

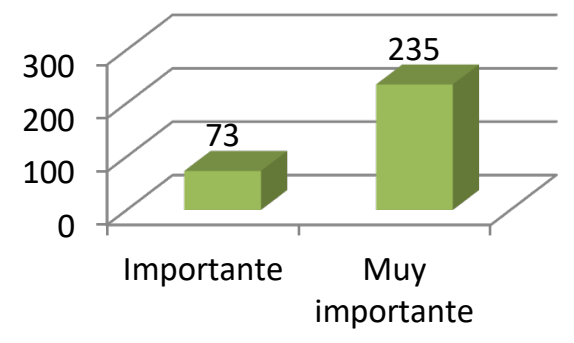

En el Gráfico 19 se hace evidente que la mayoría $(76.3 \%)$ considera muy importante contar con un guía que explique las tradiciones, costumbres e historia del lugar, con el fin de profundizar en el aprendizaje de la cultura de la comunidad visitada 20.- ¿Cuál es el monto máximo que estaría dispuesto a pagar por un programa que incluya las actividades anteriormente mencionadas?

Tabla 20: Precio

\begin{tabular}{lc}
\hline PRECIO & TOTAL \\
\hline$\$ 0-\$ 50$ & 110 \\
$\$ 100 \$ 120$ & 40 \\
$\$ 51-\$ 100$ & 158 \\
Total & $\mathbf{3 0 8}$ \\
\hline
\end{tabular}

Figura 19: Precio

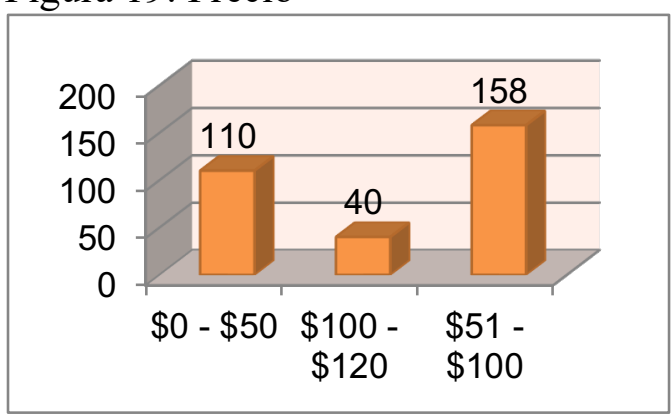

La información acerca de los precios en los cuales incurrieron los consultados que estarían dispuestos a pagar por un programa que incluya las actividades mencionadas se registró en la variable que opina de los precios. En el Gráfico 20 se visualiza el monto a pagar por el programa.

\section{Discusión}

Gracias a la investigación de mercado nos enfocaremos a aquellas personas residentes en la ciudad de Guayaquil que posean entre 26-30 años de edad al igual que los mayores de 51 años dispuestos a gastar de $\$ 50$ a $\$ 100$ en sus viajes explotando los principales atributos que atraen a los turistas de esta categoría los 
cuales que son la playa y tranquilidad que ofrece el sector.

\section{Conclusiones}

El diagnostico situacional elaborado a la parroquia Chanduy, permitió conocer y determinar las principales características de la zona donde se va a ubicar el complejo turístico. Aquí se pudo analizar aspectos importantes como: historia, como nace el nombre de esta parroquia, geografía, demografía y sobre todo se pudo conocer las principales actividades económicas y sociales del medio, todos estos datos fueron de gran importancia para realizar los siguientes pasos del proyecto. Se logró determinar que esta parroquia esta apta y cuenta con un gran potencial turístico y económico como para implementar el complejo turístico.

Las bases teóricas - científicas desarrolladas proporcionaron información suficientes sobre la investigación del tema propuesto, lo que permitió sustentar científicamente cada una de las fases del proyecto diseñado. El estudio de Mercado permitió demostrar la existencia de un mercado potencial insatisfecho, frente a la oferta actual, determinando de esta manera la viabilidad del proyecto desde el punto de vista del tamaño del mercado.

A través del estudio técnico se pudo determinar los requerimientos como infraestructura en edificaciones para las habitaciones, oficinas administrativas, áreas deportivas y complementos de ingeniería civil que el proyecto requiere de acuerdo a los productos y servicios que éste ofrece, a más de equipos y maquinaria requeridos.
El estudio financiero permitió determinar la viabilidad del proyecto, mediante la aplicación de indicadores financieros. Dentro del Estudio Organizacional se pudo establecer el tipo de empresa al que pertenecerá el centro turístico, su misión, visión, valores y de la misma manera se estableció las funciones de cada uno de los miembros de la organización. Por último en el estudio de impactos se logró determinar los efectos positivos y negativos que surgirán al implantar la empresa. El impacto ambiental es un poco preocupante debido a que en el análisis se obtuvo u bajo positivo. para la operación del proyecto.

\section{Referencias}

ANDES. (21 de Mayo de 2015). Agencia Publica de Noticias del Ecuador y Suramerica ANDES. Recuperado el 20 de Mayo de 2016, de http://www.andes.info.ec/es/noticias/ec uador-proyecta-convertir-turismoprimera-fuente-ingresos-no-petroleros2018.html Chanduy, G. P. (2014). Chanduy G.A.D Parroquial. (Ecuanegos, Productor) Recuperado el 10 de Julio de 2015, de http://chanduy.gob.ec/index.php/201212-20-22-55-35/cultura

Chila, F. (Junio de 2012). Parroquia Chanduy. Recuperado el 13 de Julio de 2015, de http://parroquiaancestralchanduy.blogsp ot.com/

Ecuador en Cifras. (2015). Ecuador en cifras. Recuperado el 02 de Agosto de 2015, de Fasciculo Provincial Guayas: http://www.ecuadorencifras.gob.ec/wpcontent/descargas/Manu- 
lateral/Resultados-

provinciales/guayas.pdf

Federación Plurinacional de Turismo

Comunitario del Ecuador. (2012).

Pangea Sostenible. Recuperado el 22 de

07 de 2015, de

http://pangeasostenible.org/2011/12/fed

eracion-plurinacional-de-turismo-

comunitario-del-ecuador/

Guillen Muñoz, E. L. (10 de Febrero de 2015). Plan estrategico turistico para la cabecera parroquial de Chanduy del canton Santa Elena, Provincia de Santa elena, año 2015. La Liberta, Santa Elena, Ecuador.

INEC. (2015). Instituto Nacional de Estadisticas y Censos. Gobierno Autonomo Descentralizado Parroquial de Chanduy, 40-41.

INEC. (2015). Instituto Naconal de Estadísticas y Censos. Recuperado el 13 de 07 de 2015, de Gobierno Nacional de la República del Ecuador: http://www.ecuadorencifras.gob.ec/ Litben, S. G. (abril de 2011). Complejo Cultural Real Alto. Obtenido de http://www.complejoculturalrealalto.org /p/publicaciones.html

MIES. (Abril de 27 de 2015). Ministerio de Inclusion Economica y Social, MIES. Recuperado el Julio de 20 de 2015, de http://www.inclusion.gob.ec/viceministr a-maldonado-expuso-sobre-politicas-yprogramas-de-inclusion-economica-ysocial-en-la-asamblea-nacional/

Ministerio del Turismo, M. (2015). Boletin de estadísticas turísticas. Recuperado el 01 de Agosto de 2016, de http://servicios.turismo.gob.ec/descarga s/Turismocifras/BoletinesEstadisticos/Anuario/Bo
letin-Estadisticas-Turisticas-2010-

2014.pdf

Mintur. (28 de Abril de 2015).

Recuperado el 2015 de Julio de 13, de http://www.turismo.gob.ec/ministeriode-turismo-tratara-las-posibilidades-dedesarrollo-del-mintur/

Mintur. (Marzo de 2015). Ministerio de Turismo del Ecuador. Recuperado el 20 de Mayo de 2016, de Coordinacion General de Estadistica e Investigacion: www.quito-

turismo.gob.ec/index.php/es/estadisticas /quito-en-cifras?download

OMT, O. M. (2015). World Tourism Organization UNWOT. Recuperado el 2015 de Julio de 12, de http://www2.unwto.org/es/content/porque-el-turismo

Secretaría Nacional de Planificación y Desarrollo, S. (2014). Plan Nacional del Buen vivir 2013 - 2017. Quito, Pichincha, Ecuador: EL telégrafo.

Sión de Josse, E. (19 de Marzo de 2011). Ministerio de Trurismo. Recuperado el 02 de Agosto de 2015, de http://www.turismo.gob.ec/wpcontent/uploads/2015/04/REGLAMEN TO-CENTROS-TURISTICOSCOMUNITARIOS.pdf

Turismo, M. d. (09 de Enero de 2011). Reglamento Oficial de establecimientos hoteleros. Guayaquil, Guayas, Ecuador. UNWTO. (2015). Resultados regionales del turismo. Panorama OMT del turismo internacional, 1-16.

Vargas, M. R., Fuentes, N. M., \& Ocaña, A. O. (2014). "La gestión del turismo comunitario en el marco de la economía popular y solidaria en Ecuador". Revista Turydes: Turismo y Desarrollo, 17. 
Propuesta de un complejo turístico en la Parroquia Chanduy, Provincia de Santa Elena 